\title{
Vorwort zur ersten Auflage
}

Dieses Repetitorium soll in kompakter Form einführende Lehrveranstaltungen in das Studienfach Wirtschaftsinformatik begleiten. Als Studierender werden Sie in kurzer, prägnanter Form mit den wesentlichen Inhalten aus der Vorlesung vertraut gemacht. Dabei ersetzt das Repetitorium kein Lehrbuch, das tiefer und facettenreicher den Stoff der Wirtschaftsinformatik aufbereitet. Aus diesem Grund finden Sie auch viele Literaturverweise auf wichtige und bewährte Standardwerke zur Einführung in die Wirtschaftsinformatik.

Wir haben uns bemüht, den Stoff anhand einer durchgängigen Fallstudie greifbar und nachvollziehbar zu machen. Zu Beginn jedes Kapitels wird der relevante Ausschnitt der Fallstudie für den im Kapitel behandelten fachlichen Inhalt vorgestellt. Daran schließt sich die Kurzdarstellung der Theorie an. Das Kapitel schließt mit wissensortientierten Detailfragen zur Theorie und problemlösungsorientierten Komplexfragen, die sich auf die Fallstudie beziehen. Für alle Fragen finden Sie im Anhang unsere Musterlösungen. Allerdings erwarten wir, dass Sie sich auch eigenständig mit den Fragen auseinandersetzen. Das ist zwar mühsam, sichert aber den nachhaltigen Lernerfolg.

Für die aktive Mitarbeit an der Ausarbeitung der Fallstudie bedanken wir uns bei Dipl.-Wirtschaftsinformatiker (BA) Stefan Brey, Dipl.-Wirtschaftsinformatiker (BA) Michael Haas, Dipl.-Wirtschaftsinformatikerin (BA) Carina Maucher, Dipl.-Wirtschaftsinformatiker (BA) Fabian Schneider, Dipl.-Wirtschaftsinformatiker (BA) Peter Raisch und Dipl.-Wirtschaftsinformatiker (BA) Stefan Rietzler.

Natürlich gehen alle Fehler inhaltlicher und formaler Art nur zu unseren Lasten. Schreiben Sie uns also, wenn Sie Fehler finden oder Verbesserungsvorschläge haben.

Und nun wünschen wir Ihnen viel Spaß mit einem faszinierenden Studienfach der Wirtschaftsinformatik!

Ravensburg und Kempten, April 2007 
